\title{
Huge broadening of the crystal-fluid interface for sedimenting colloids
}

\author{
Elshad Allahyarov ${ }^{1,2,3}$, Hartmut Löwen ${ }^{1}$ \\ (1) Institut für Theoretische Physik, \\ Heinrich-Heine-Universität Düsseldorf, D-40225 Düsseldorf, Germany \\ (2) Joint Institute for High Temperatures, \\ Russian Academy of Sciences, Izhorskaya 13/19 , 117419 Moscow, Russia \\ (3) Department of Physics, Case Western Reserve University, Cleveland, 44106 Ohio, USA
}

\begin{abstract}
For sedimenting colloidal hard spheres, the propagation and broadening of the crystal-fluid interface is studied by Brownian dynamics computer simulations of an initially homogeneous sample. Two different types of interface broadenings are observed: the first occurs during growth and is correlated with the interface velocity, the second is concomitant with the splitting of the crystalfluid interface into the crystal-amorphous and amorphous-liquid interfaces. The latter width is strongly peaked as a function of the gravitational driving strength with a huge amplitude relative to its equilibrium counterpart.
\end{abstract}

PACS numbers: 82.70.Dd,61.20.Qg,87.15.A 


\section{INTRODUCTION}

Sedimentation of colloidal particles in a liquid is a widespread phenomenon which governs the formation of river sediments close to salty sea water [1], controls the function of red blood cells [2], and is technologically used to separate different sorts of particles by centrifugation [3]. Gravity is also used to compactify colloidal samples. In a finite container, the actual settling process reaches a sedimentation-diffusion equilibrium, which is characterized by a static colloidal density profile. Much of our particle-resolved knowledge of dense suspensions stems from model hard sphere colloidal dispersions made up of sterically-stabilized particles. In equilibrium, it has been shown that density profiles contain the isothermal equation of state of the hard sphere system [4, 5]. At high gravitational strengths (or Peclet numbers) the density of hard spheres at the bottom of suspension increases above the lower limit of the solid-liquid coexistence $\phi=0.492$ [6], and a crystallization in the bottom layers occurs [7 -9 ]. The number of crystalline layers is controlled by gravity and by the sedimentation velocity of particles, and the overall particle density per area. While the equilibrium properties of crystal sediments are well-explored by now, the dynamics and relaxation towards equilibrium is by far less understood [10, 11]. In particular, it is known that crystallization occurs under gravity at different conditions than in the bulk [12, 13]. The technique of colloidal templating has been used to steer colloidal crystallization layer-by-layer in gravity «14, 15] and the kinetics of crystalline defects [16, 17] has been debated for sediments.

Using real-space confocal microscopy techniques, Dullens and coworkers [18] have recently measured a dynamical broadening of the hard sphere solid-fluid interface during sedimentation. This points to the essential role of crystal nucleation in the supersaturated fluid and the subsequent built-in of "crystal packages" into the interface. In a very slowly growing interface, and at low Peclet numbers, a broadening of the fluid-solid interfacial width relative to its equilibrium value was found. In this letter, we address the broadening of the solid-fluid hard sphere interface in the sedimentation process by extensive Brownian dynamics computer simulations. We find two different types of broadenings of the fluid-crystal interface: the first occurs during growth and is correlated with the interface velocity. This type of broadening is not driven by gravity as it also shows up in the gravity-free case [19]. The second type of interface broadening occurs before the splitting of the crystal- amorphous interface from the amorphous-liquid interface. The separated interface includes a region 


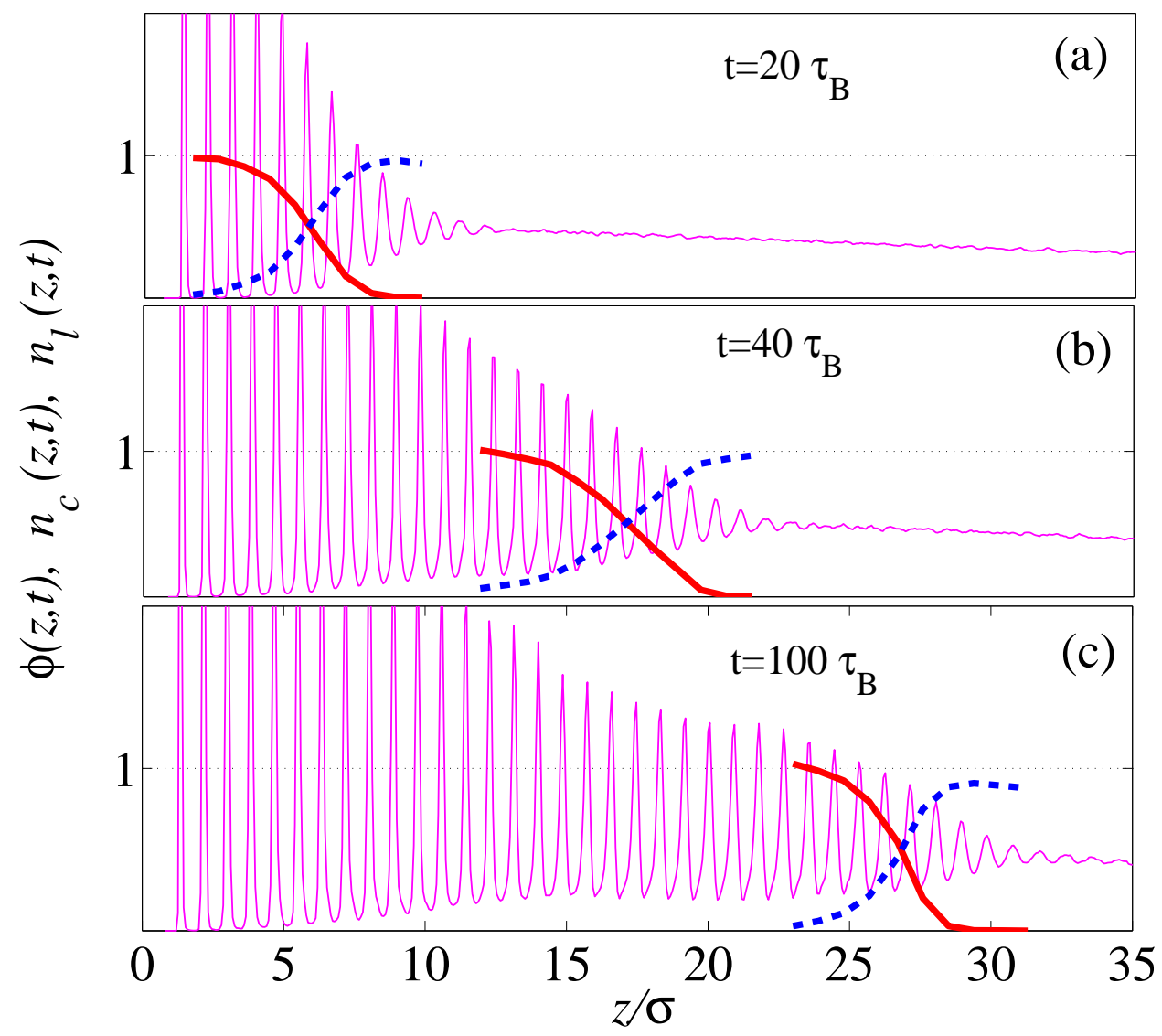

FIG. 1. Laterally averaged packing fractions $\phi(z, t)$ (thin pink lines) versus reduced height $z / \sigma$ for three different times (a-c): $t=20 \tau_{B}, 40 \tau_{B}, 100 \tau_{B}$. The parameters are $P e=0.5, \eta_{A}=45.4$, and $\phi=0.3$. Also shown are the interfacial profiles $n_{c}(z, t)$ for the "crystalline" particles (thick red line) and $n_{l}(z, t)$ for the "liquid-like" particles (dashed blue line). The interface position $z_{0}(t)$ is determined from the crossing point of the thick red and dashed blue lines.

which is structurally disordered and dynamically arrested. The latter width behaves nonmonotonic in the gravitational driving strength (or Peclet number $P e$ ) and exhibits a huge peak relative to its equilibrium counterpart. While the first type of broadening has not yet accessed in confocal microscopy, the latter is consistent with the measurements of Dullens et al [18]. However, their measurements were only performed for low Peclet numbers before the splitting of two interfaces happen. 


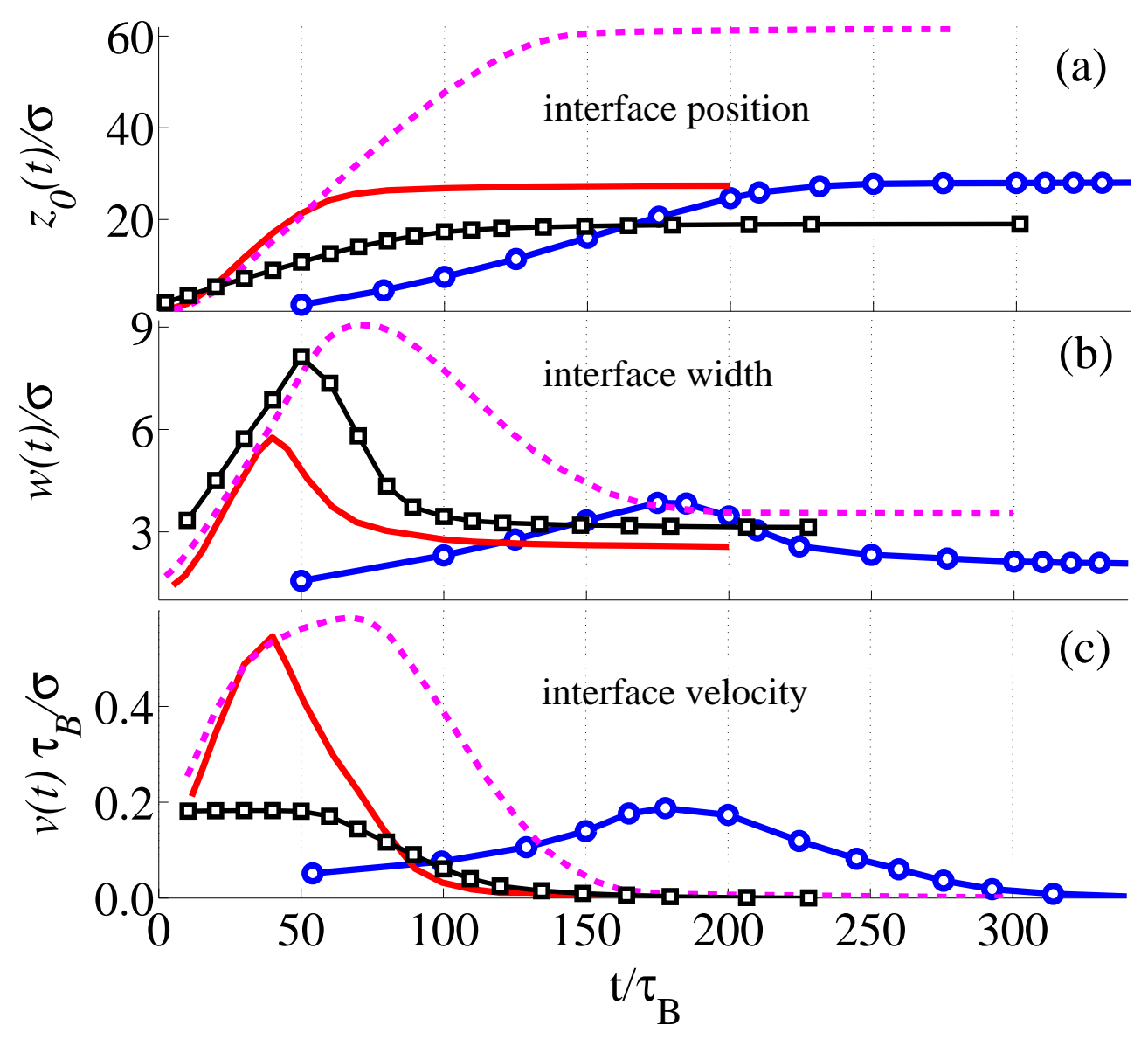

FIG. 2. Reduced interface position $z_{0}(t) / \sigma(\mathrm{a})$, reduced interfacial width $w(t) / \sigma(\mathrm{b})$, and reduced propagation velocity $v(t) \tau_{B} / \sigma(\mathrm{c})$ as a function of reduced time $t / \tau_{B}$. Full blue lines with circles are for $\eta_{A}=45.4$ and $\phi=0.1$, full red lines are for $\eta_{A}=45.4$ and $\phi=0.3$, dashed pink lines are for $\eta_{A}=90.8$ and $\phi=0.3$. Full black lines with squares are simulation data for the gravity-free $(P e=0)$ suspension with a volume fraction $\phi=0.51$ and $\eta_{A}=46.27$.

\section{SIMULATION AND ANALYSIS}

In our Brownian dynamics computer simulations, we use an adapted code for hard spheres [20] of diameter $\sigma$, where the short-time infinite-dilution diffusion coefficient $D_{0}$ sets the Brownian time scale $\tau_{\mathrm{B}}=\sigma^{2} / D_{0}$. A time step of $\Delta t=0.001 \tau_{\mathrm{B}}$ was used in integrating the stochastic equations of motion. The simulation box contains $N$ hard spheres and has a rectangular shape with dimensions $L_{\mathrm{x}}=40.8 \sigma, L_{\mathrm{y}}=43.2 \sigma$, and $L_{\mathrm{z}}$ varied between $54 \sigma$ and 
$240 \sigma$. Various systems with a packing fraction $\phi=\frac{\pi \sigma^{3}}{6} \frac{N}{L_{\mathrm{x}} L_{\mathrm{y}} L_{\mathrm{z}}}$ in the range $0.1 \leq \phi \leq 0.45$ were investigated. A further important system parameter is the gravitational load, or the surface (or areal) density of particles $\rho_{A}=N / L_{\mathrm{x}} L_{\mathrm{y}}$, which we scale to

$$
\eta_{A}=N \sigma^{2} / L_{\mathrm{x}} L_{\mathrm{y}}=\phi \frac{6 L_{\mathrm{z}}}{\pi \sigma}
$$

Thus different combinations of $\phi$ and $L_{\mathrm{z}}$ result in the same $\eta_{A}$. Simulations were carried for $\eta_{A}=45.4$ with $N=80000$ particles and for $\eta_{A}=90.8$ with $N=160000$ particles. The quantity $\eta_{A}$ is chosen large enough to produce a crystal-fluid interface at a given gravitational strength $P e$. The gravity acceleration $g$ points along the $-z$-direction and has a relative strength

$$
P e=m g \sigma /\left(2 k_{B} T\right)
$$

where $k_{B} T$ denotes the thermal energy and $m$ the buoyant mass of the colloidal particles [21]. Periodic boundary conditions are employed in the $x$ and $y$ directions, while two hard walls are placed at $z=0$ and $z=L_{z}$. Next to the wall at $z=0$ we place a triangular layer of fixed spheres with a lattice constant $a=1.133 \sigma$ which acts as an initial template for crystal growth [22]. Without a template the crystallization happens in a few bottom layers containing small grains, large defects and fault stackings. This strongly suppresses the formation and following upward propagation of a single-phase crystalline front along the sediment.

All simulations were started from an initial configuration with a homogeneous distribution of colloids in the simulation box except the template particles in the seed layer next to the bottom wall. This mimics an initially stirred solution to which gravity is applied instantaneously [11]. A total simulation time of $500 \tau_{\mathrm{B}}$ was accessed during our simulations. Within this time each particle can sediment about $1000 \sigma P e$, a distance at least 5 times larger than the characteristic sedimentation length $h \approx H \times 0.55 / \phi$. Here $H$ is the hight of the sedimented segment above which the packing fraction of suspension is $\phi<0.5$. We averaged our final stage and time-independent results over 20 different initial configurations in order to improve the statistics.

For subsequent times $t$, we calculated the laterally averaged one-particle packing fraction which is defined as

$$
\phi(z, t)=\frac{\pi}{6} \frac{\sigma^{3}}{L_{\mathrm{x}} L_{\mathrm{y}}} \iint d x d y \rho(x, y, z, t)
$$




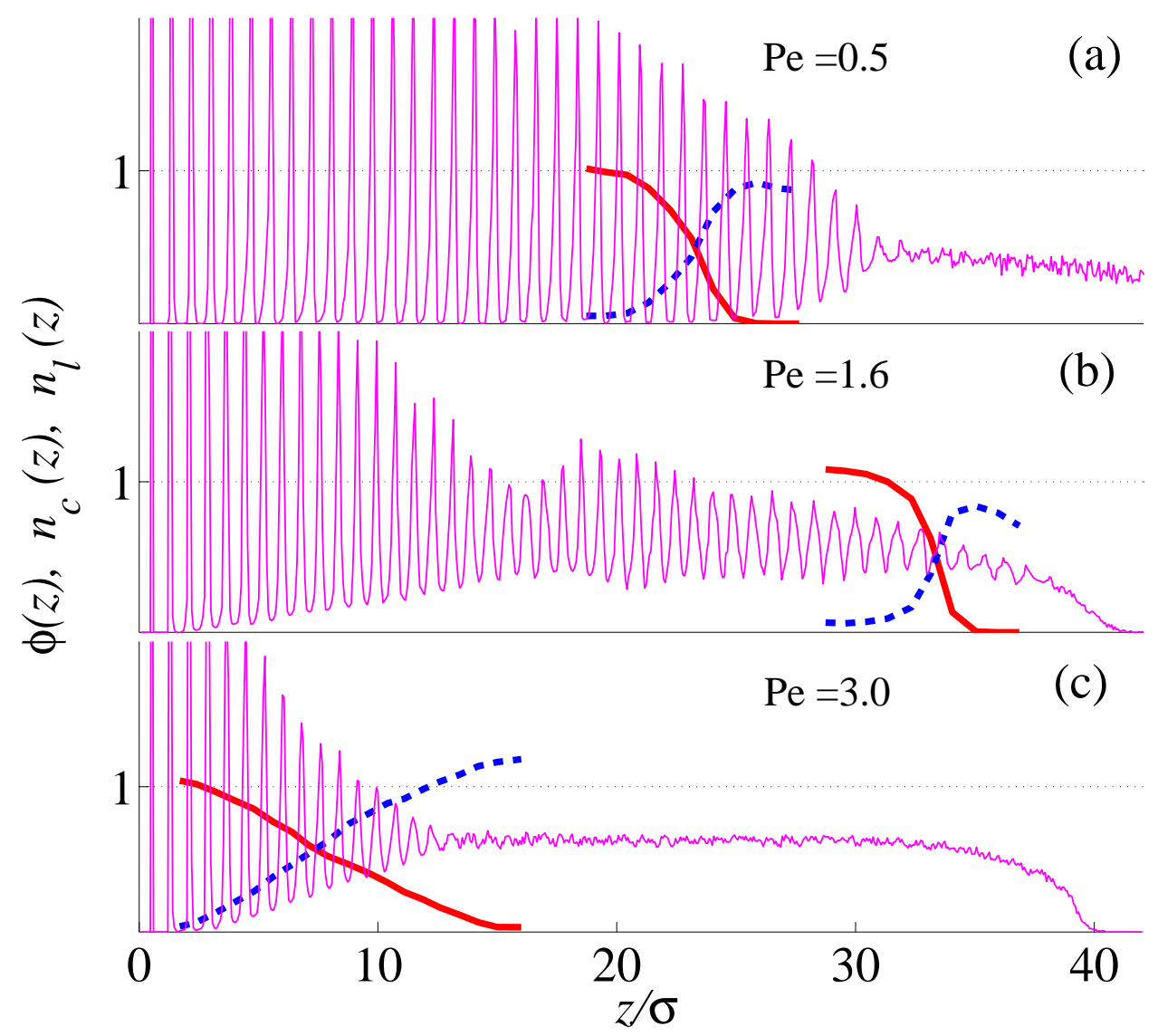

FIG. 3. Same as Figure 1, but now for a fixed large time $t=t_{l}=500 \tau_{B}$ and three different Peclet numbers: (a) $P e=0.5$, (b) $P e=1.6$, (c) $P e=3.0$ at $\eta_{A}=45.4$ and $\phi=0.1$.

and is resolved along the vertical $z$-coordinate. Here $\rho(x, y, z, t)$ is the local one-body density of particles at a given time $t$. We further identified "crystalline" particles with a crystallike surrounding according to a commonly used criterion [23]: the local orientational-order parameter $\vec{q}_{6}(i)$ is calculated for each particle $i[24,25]$. When two particles $i$ and $j$ are separated by $r \leq 1.3 \sigma$, we associate a crystalline bond to these particles, if $\vec{q}_{6}(i) \cdot \vec{q}_{6}(j)>0.5$. A particle which has at least 8 of these bonds is considered to be crystalline. All other particles are identified as a "liquid-like" particles. The corresponding local packing fractions of crystalline particles $n_{c}(z, t)$ and liquid particles $n_{l}(z, t)$ are also calculated and give rise to a $q_{6}$ interface, an example of which is given in Figure 1. We now define the interface position $z_{0}(t)$ by the implicit condition $n_{c}\left(z_{0}(t), t\right)=n_{l}\left(z_{0}(t), t\right)$, i.e. by the position where 
the fraction of crystalline-particles equals that of the liquid-like particles. The interfacial width $w(t)$ is defined as the inverse of the maximal slope of the $n_{c}$ profiles

$$
w(t)=\left.\left(\frac{\partial n_{c}(z, t)}{\partial z}\right)^{-1}\right|_{z=z_{0}(t)}
$$

We finally define the propagation velocity of the solid-fluid interface as $v(t)=d z_{0}(t) / d t$.

\section{MECHANISM 1: KINETIC BROADENING}

Results for the interfacial profiles at three different times are shown in Figure 1. Clearly the crystal-fluid interface is propagating from the bottom of the container (left side in the figure) into the fluid. The lowering of peak heights in the density profile of Figure 1te in the region between $z=14 \sigma$ and $z=27 \sigma$ originates from the stacking faults [9, 26] and defects introduced by a small amount of rhcp and hcp crystalline grains. These defects tend to dissipate into fcc crystalline layers during longer time simulations. The system size also plays a crucial role in the dissipation of stacking defects [27]. When the crystal grows, the interface is getting broader. This is clearly illustrated in Figure 2 where the interface position $z_{0}(t)$, its width $w(t)$ and its propagation velocity $v(t)$ are shown simultaneously. The interface velocity is non-monotonic in time: it first gets accelerated, reaches a maximum and decays to zero. The initial acceleration has to do with an "induction time" to get particles into positions which are structurally favorable for subsequent crystal growth. The final slowingdown is due to the approach to equilibrium where ideally a finite height of the interface is reached. Concomitantly with the non-monotonic behavior of the interface velocity, there is a broadening of the interface: as a function of time, it first broadens and then shrinks again. The saturation value at large times, however, is significantly larger than the initial width. A direct comparison of Figure $2 \mathrm{~b}$ and $2 \mathrm{c}$ reveals that the maximum of the propagation velocity coincides with the maximal interfacial width demonstrating that these two phenomena are correlated. The effect is stable for different overall densities $\eta_{A}$. An increased $\eta_{A}$ just retards the occurrence of the velocity and width maximum such that they occur at larger simulation times. A similar retardation effect takes place in systems with low packing fraction $\phi$ at a fixed areal density $\eta_{A}$, compare solid lines with and without symbols in Figure 2.

As a first type of interfacial widening, we therefore identify a kinetic broadening of the interface: upon growth, the $q_{6}$-interface becomes less steep, see thick lines in Figure $1 \mathrm{~b}$. 
This has to do with the fact that the system has less time to structurally arrange when the interface propagates quickly. An additional simulation for zero gravity revealed that the kinetic broadening effect exists also at zero Peclet number [28]. However, in the gravityfree case the front growth velocity has no maximum. As a reference, simulation data for the front position, interfacial width and the propagation velocity for a coexisting fluid and crystal [23, 29, 30] in a gravity-free system are included into Figure 2 as full lines with squares.

\section{MECHANISM 2: INTERFACE SPLITTING}

We now address the crystal-fluid interface in the final state of the sediment after a long time $t_{l}=500 \tau_{\mathrm{B}}$ and discuss its properties as a function of the Peclet number (at fixed $\left.\eta_{A}\right)$. In Figure 3 , the laterally-averaged packing fraction profiles are shown at $t_{l}$ for three different Peclet numbers $P e=0.5,1.6,3.0$. Interestingly, the position of the interface (as visualized by the crossing of the red and blue lines) behaves non-monotonic in the Peclet number, see also Figure 4, which contradicts the expectation based on equilibrium arguments. This expectation is based on a discussion of the two limiting cases $P e \rightarrow 0$ and $P e \rightarrow \infty$ in equilibrium: for zero gravity, the density profile is homogeneously distributed over the full simulation box (apart from local density correlations at the hard wall) with a low bulk volume fraction. Hence the solid-fluid interface (if at all) is close to the bottom wall. In the opposite limit $P e \rightarrow \infty$, there is a finite number of layers with a closed packed density which then abruptly drops to a vacuum at higher $P e$ at a height of about $30 \sigma$. Interpolating between these two limiting cases, in equilibrium, the interface position increases with Peclet number $P e$. Starting from $P e \approx 0.8$ the single crystalline-liquid interface splits into two separate interfaces, the crystalline-amorphous and the amorphous-liquid interfaces. When the gravitational load increases, the amount of amorphous glass grows partly from the sedimented liquid particles, and partly from the collapsed crystalline layers. Higher loads

completely destroy the crystal leaving only a few intact layers. Besides of the $q_{6}$-interface position, a position of the amorphous-liquid interface can be defined at the height where the coarse-grained density (averaged over the oscillations) [31] equals the bulk coexisting fluid volume fraction of 0.492 . As opposed to the nonmonotonic variation of the $q_{6}$-interface position, the position of the amorphous-liquid interface is monotonic, see Figure 4. While 

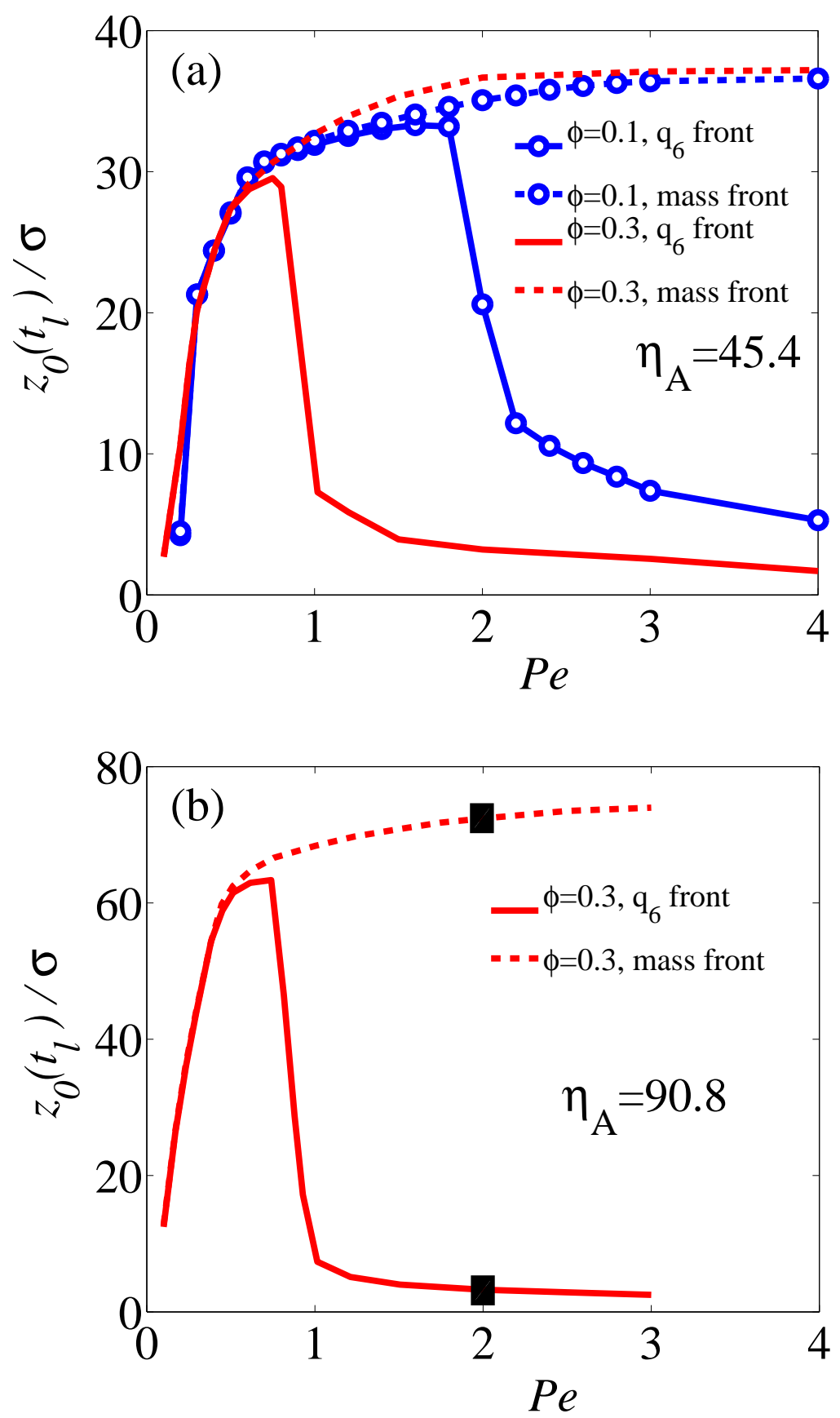

FIG. 4. (a) Position $z_{0}\left(t_{l}\right) / \sigma$ of the $q_{6}$-interface (full lines), and of the amorphous-liquid interface (dashed lines) versus Peclet number $P e$ for two areal densities $\eta_{A}=45.4$ (a) and $\eta_{A}=90.8$ (b) at a fixed large time $t=t_{l}=500 \tau_{B}$. The interface splitting is clearly visible. Black squares at $P e=2$ correspond to the amorphous-liquid and $q_{6}$-interfaces analyzed in Figure 5 ,

the positions of the $q_{6}$-interface and the amorphous-liquid interface coincide for small Peclet 


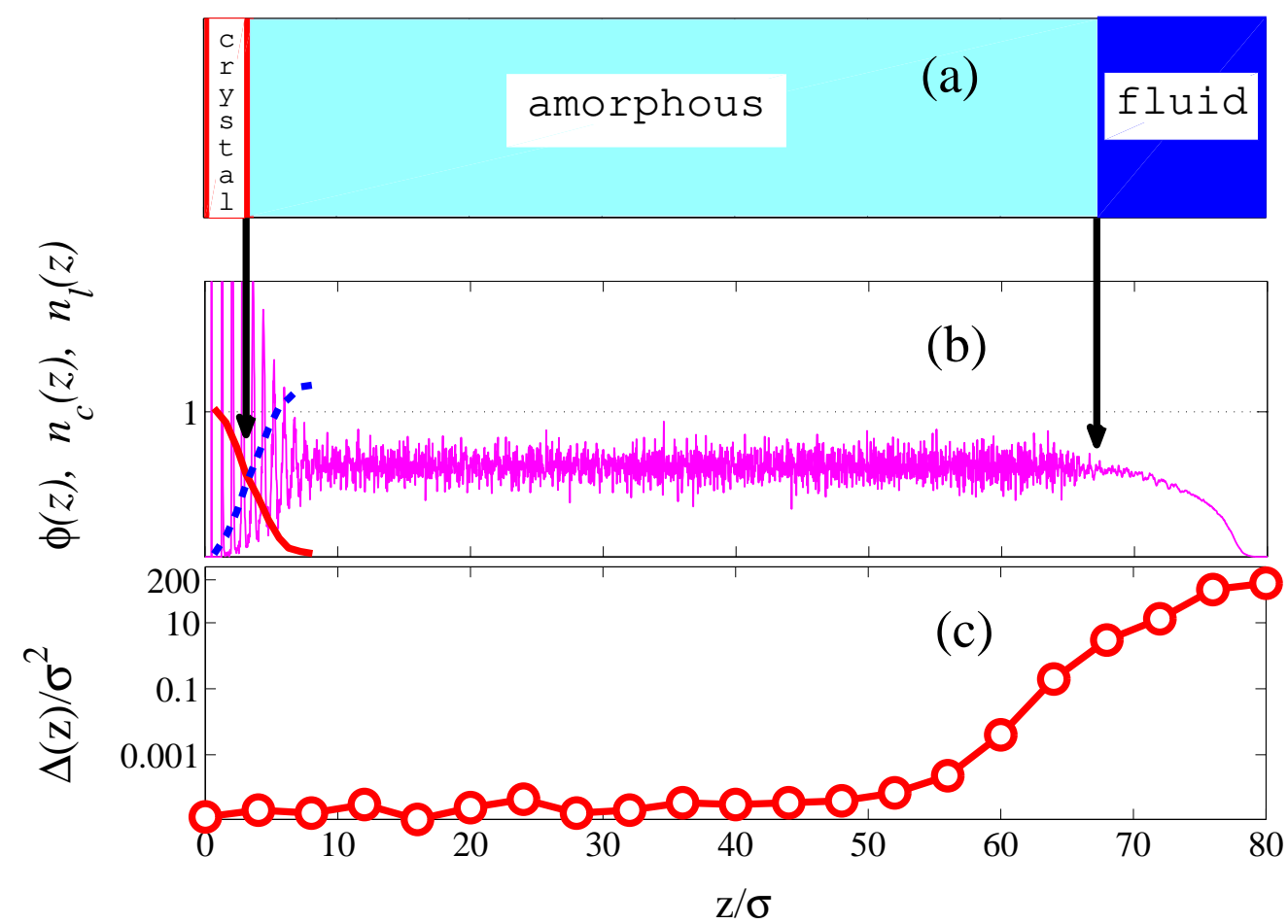

FIG. 5. Simulation results for the sediment at $t=t_{l}=500 \tau_{B}$ for system parameters $P e=2$, $\phi=0.3$ and $\eta_{A}=90.8$. (a) Schematic picture showing the crystalline, amorphous and fluid zones in the sediment. (b) Laterally averaged packing fraction $\phi(z)$ (thin pink lines), interfacial profile $n_{c}(z)$ for the "crystalline" particles (thick red line), interfacial profile $n_{l}(z)$ for the "liquid-like" particles (dashed blue line) versus reduced height $z / \sigma$. (c) Mean square displacement $\Delta(z)$ of the particles along the sediment obtained within a time window of $\Delta t=10 \tau_{B}$. The positions of the crystal-glass and glass-fluid interfaces correspond to the black squares in Figure 4b.

number, there is a splitting above a threshold leading to the non-monotonic behavior. The non-monotonicity can be explained by the formation of a dynamically arrested region between the splitted $q_{6}$ and amorphous-liquid interfaces schematically shown in Figure 5 a for a system with $\phi=0.3, \eta_{A}=90.8$ and $P e=2$. Within the glassy amorphous region the particles are structurally disordered, see Figure 5b, and dynamically caged such that they cannot find suitable surrounding to nucleate into a big, and layered, crystal. This is documented by the smallness of the mean square displacement of particles in the amorphous layer, see Figure 5r. The amorphous dynamically arrested part stops the propagation of the $q_{6}$-interface. Strong gravity acts therefore like a fast and deep compression, a situation 
which favours glass formation in general. The disordered region does not seem to be composed of polycrystalline material as this would have resulted in a finite fraction of crystalline particles. As shown in Figure 4 a and $4 \mathrm{~b}$, the Peclet number threshold for splitting decreases for increasing packing fraction $\phi$ at fixed $\eta_{A}$, but does not depend on the imposed overall density $\eta_{A}$ for fixed $\phi$. Closely above the Peclet number threshold at which interface splitting occurs, the width $w$ exhibits a strong peak, see Figure 6. In fact, the width increases by an order of magnitude relative to its low-Peclet-number value and then decreases again. This surprising huge broadening points to an extremely broad interface between the initial crystalline layers and the subsequently formed disordered material. The subsequent decrease for higher Peclet number is qualitatively understood by considering the limit $P e \rightarrow \infty$ : if everything is dominated by strong gravity, the interface then is expected to sharpen due to enforced crystallization.

We now compare our results to the real-space experiment by Dullens and coworkers [18]. The broadening observed in Ref. [18] was measured for long times at relatively small Peclet numbers (smaller than 0.8) and for larger gravitational loads $\eta_{A}$ than considered in our work. The interfacial velocity was small but nonzero. This implies that the broadening observed in [18] is consistent with our findings at long times for low Pe, before the interface splitting occurs. It would be interesting to do further experiments at higher $P e$ to verify the interface splitting and the huge interface broadening predicted by our simulations.

\section{CONCLUSION}

In conclusion, we have studied the width of a crystal-fluid interface in a sedimenting suspension of hard spheres by Brownian dynamics computer simulations. Two qualitatively different types of broadening are observed. The first type of interfacial broadening is purely kinetic. It is not triggered by gravity but just correlates with the interface velocity. The second type of broadening is almost static and is huge if the structural crystallinity interface and the amorphous-liquid interface split. Between these two interfaces, an amorphous dense sediment is formed which exhibits a wide structural interface with the lower crystalline part of the sample. This prediction can in principle be verified by real-space experiments [18, 23]. It might also be important for granulates [32] where the Peclet number is high, and for crystallization in complex plasma [33]. 


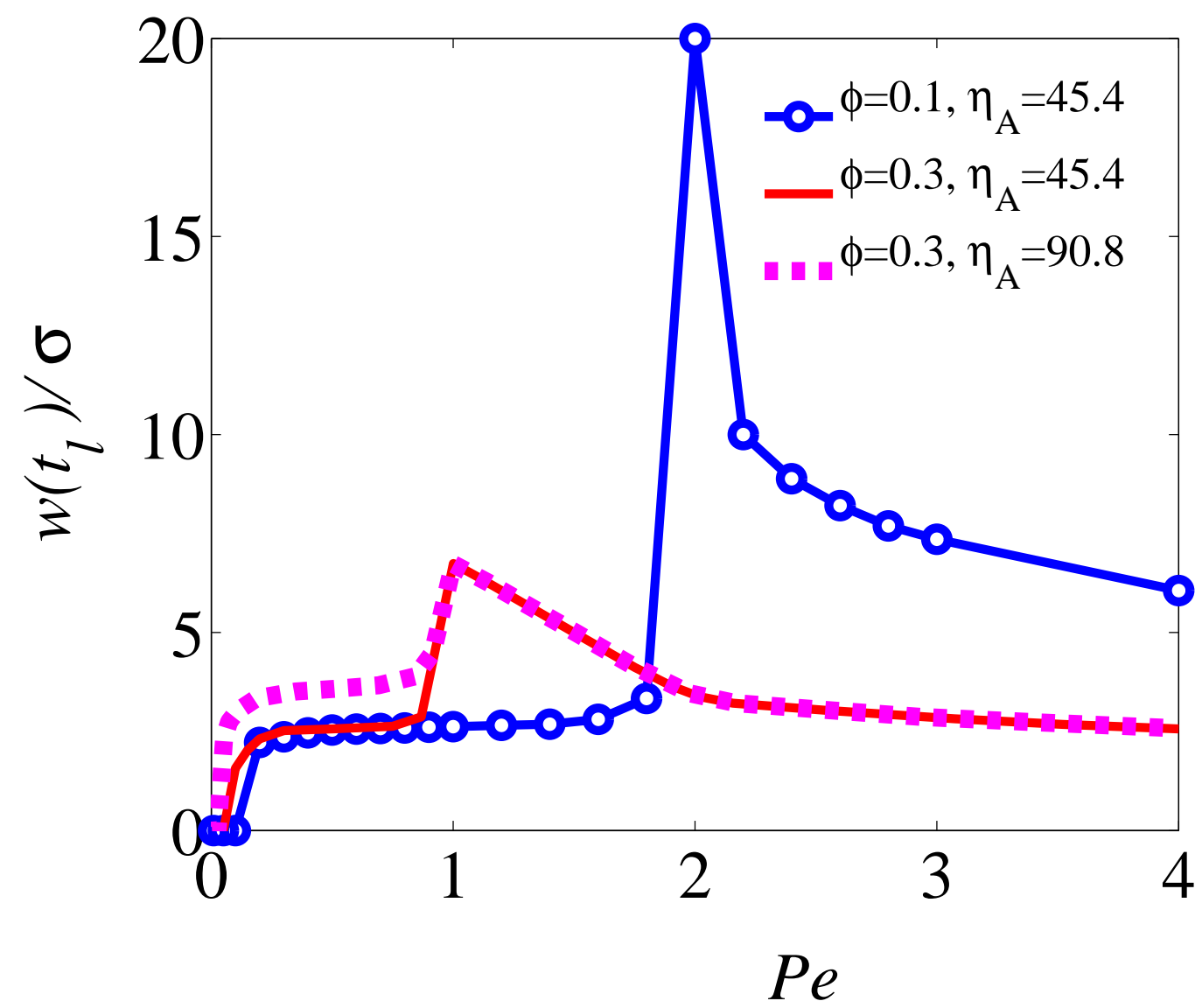

FIG. 6. Interfacial width $w\left(t_{l}\right) / \sigma$ versus Peclet number $P e$ at a fixed large time $t=t_{l}=500 \tau_{B}$ for two different packing fractions $\phi=0.1$ (line with symbols) and $\phi=0.3$ (full line) at fixed areal density $\eta_{A}=45.4$, and for packing fraction $\phi=0.3$ and $\eta_{A}=90.8$ (dashed line).

Real experimental samples possess an intrinsic polydispersity. Furthermore there are solvent-mediated hydrodynamic interactions. Most of the influence of hydrodynamic interactions can be captures by an effective short-time diffusion constant $D_{0}$. Nevertheless the influence of polydispersity [23] and hydrodynamic interactions [11, 34, 35] on crystal growth remains for future exploration. Furthermore new effects are expected for attractive systems [36] as witnessed by different dynamically arrested states (attractive glasses) [37] which could impede crystallization during sedimentation. The latter case is realized for colloid-polymer mixtures.

It is worth to mention that some of the present findings on the interface broadenings may be characteristics of fcc $\{111\}$ growth from a patterned template with triangular lattice 
considered in current study. It will be useful to check whether a similar broadenings takes

place in the $\{100\}$ growth, a case considered in Refs. [29, 30] for gravitation-free hard sphere systems.

In general, our findings can be used to steer the thickness of crystalline and amorphous layers as well as their interfacial structure by sedimentation. This may help to open the way for designing smart colloidal materials with new optical and rheological properties.

\section{ACKNOWLEDGMENTS}

We thank R. Dullens, M. Marechal, S. U. Egelhaaf and K. Sandormirski for helpful discussions. Financial support from the DFG within SPP 1296 is gratefully acknowledged. E.A. also acknowledges partial support of this work by the US Department of Energy under grant DE-FG02-05ER46244.

[1] J. J. Naudin, G. Cauwet, M. J. Chretiennot-Dinet, B. Deniaux, J. L. Devenon, and H. Pauc, Estuarine Coastal and Shelf Science 45, 303 (1997).

[2] H. Walter and P. A. Albertsson, Experimental Cell Research 67, 218 (1971).

[3] A. P. Philipse, Current Opinion in Colloid. \& Interface Science 2, 200 (1997).

[4] R. Piazza, T. Bellini, and V. Degiorgio, Phys. Rev. Letters 71, 4267 (1993).

[5] T. Biben, J. P. Hansen, and J. L. Barrat, J. Chem. Phys. 98, 7330 (1993).

[6] R. L. Davidchack and B. B. Laird, J. Chem. Phys. 108, 9452 (1998).

[7] T. Biben, R. Ohnesorge, and H. Löwen, Europhys. Letters 28, 665 (1994).

[8] M. Marechal and M. Dijkstra, Phys. Rev. E 75, 061404 (2007) .

[9] A. Mori, S. Yanagiya, Y. Suzuki, T. Sawada, and K. Ito, J. Chem. Phys. 124, 174507 (2006).

[10] C. P. Royall, J. Dzubiella, M. Schmidt, and A. van Blaaderen, Phys. Rev. Letters 98, 188304 (2007).

[11] A. Wysocki, C. P. Royall, R. Winkler, G. Gompper, H. Tanaka, A. van Blaaderen, and H. Löwen, Soft Matter 5, 1340 (2009) .

[12] B. J. Ackerson, S. E. Paulin, B. Johnson, W. van Megen, and S. Underwood, Phys. Rev. E 59, 6903 (1999). 
[13] Z. D. Cheng, P. M. Chaikin, J. X. Zhu, W. B. Russel, and W. V. Meyer, Phys. Rev. Letters 88, 015501 (2002).

[14] J. P. Hoogenboom, D. Derks, P. Vergeer, and A. van Blaaderen, J. Chem. Phys. 117, 11320 (2002).

[15] I. B. Ramsteiner, K. E. Jensen, D. A. Weitz, and F. Spaepen, Phys. Rev. E 79, 011403 (2009).

[16] A. Mori, Y. Suzuki, and S. I. Yanagiya, Fluid Phase Equilibria 257, 131 (2007).

[17] J. P. Hoogenboom, P. Vergeer, and A. van Blaaderen, J. Chem. Phys. 119, 3371 (2003).

[18] R. P. A. Dullens, D. G. A. L. Aarts, and W. K. Kegel, Phys. Rev. Letters 97, 228301 (2006).

[19] This finding contrasts simple Ginzburg-Landau-theory for the interfacial dynamics of a onecomponent non-conserved order parameter profile (like the crystallinity). Under the assumption that the curvatures of the free energies in order parameter space are independent of the undercooling, the interfacial width shrinks with its propagation velocity, see e.g.: J. Bechhoefer, H. Löwen, L. S. Tuckerman, Phys. Rev. Letters 67, 1266 (1991).

[20] B. Cichocki and K. Hinsen, Physica A 166, 473 (1990).

[21] In colloidal physics and fluid mechanics the motion of a solute particle under external driving field is characterized by a Peclet number $P e=\tau_{D} / \tau_{S}$, which represents the competition between advection and diffusion processes. Here $\tau_{D}$ is the time it takes a particle to diffuse its own radius $\tau_{D}=\sigma^{2} /\left(4 D_{0}\right)$, and $\tau_{S}$ is the time it takes the particle to sediment the same distance $\tau_{S}=\sigma /\left(2 v_{S}\right)$. Taking into account the fact that the sedimentation velocity $v_{S}$ of the solute particle can be approximated by its terminal velocity under the force of gravitational drag, $v_{S}=m g / \xi$, and the diffusion coefficient of the solute particle is $D_{0}=k_{B} T / \xi$ ( $\xi$ is the Stokes friction coefficient), the Peclet number can be rewritten as: $P e=\frac{\sigma}{2} \frac{v_{S}}{D_{0}}=\frac{\sigma}{2} \frac{(m g / \xi)}{\left(k_{B} T / \xi\right)}=$ $\frac{\sigma}{2} \frac{m g}{k_{B} T}$.

[22] M. Heni and H. Löwen, Phys. Rev. Letters 85, 3668 (2000).

[23] K. Sandomirski, E. Allahyarov, H. Löwen, and S. U. Egelhaaf , Soft Matter DOI:10.1039/C1SM05346A (2011).

[24] P. J. Steinhardt, D. R. Nelson, and M. Ronchetti, Phys. Rev. B 28, 784 (1983).

[25] P. R. ten Wolde, M. J. Ruiz-Montero, and D. Frenkel, Phys. Rev. Letters 75, 2714 (1995).

[26] A. Mori, Y. Suzuki, S. I. Yanagiya, T. Sawada, and K. Ito, Mol. Phys. 105, 1377 (2006).

[27] A. Mori, J. Cryst. Growth. 318, 66 (2011).

[28] The $P e=0$ simulation was performed for $\phi=0.51$ and $\eta_{A}=46.27$ with $N=80000$ particles 
in a box of size $L_{\mathrm{x}}=40.8 \sigma, L_{\mathrm{y}}=43.2 \sigma$, and $L_{\mathrm{z}}=48.5 \sigma$. Again a template layer of fixed spheres with a triangular layer lattice constant $a=1.133 \sigma$ was placed above the bottom plate of the box.

[29] T. Zykova-Timan, J. Horbach, and K. Binder, J. Chem. Phys. 133, 014705 (2010).

[30] A. Mori, J. Phys. Soc. Jpn. 66, 1579 (1997).

[31] In detail, the coarse-grained density is obtained from the $z$-resolved profiles by integrating between two subsequent minima.

[32] J. A. Both and D. C. Hong, Phys. Rev. E 64, 061105 (2001).

[33] M. Rubin-Zuzic, G. E. Morfill, A. V. Ivlev, R. Pompl, B. A. Klumov, W. Bunk, H. M. Thomas, H. Rothermel, O. Havnes, and A. Fouqut, Nature Physics 2, 181 (2006).

[34] N. Q. Nguyen and A. J. C. Ladd, J. Fluid Mechanics 525, 73 (2005).

[35] A. Moncho-Jorda, A. A. Louis, and J. T. Padding, Phys. Rev. Letters 104, 068301 (2010).

[36] M. Leocmach, C. P. Royall, and H. Tanaka, Europhys. Letters 89, 38006 (2010).

[37] E. Zaccarelli, H. Löwen, P. P. F. Wessels, F. Sciortino, P. Tartaglia, and C. N. Likos, Phys. Rev. Letters 92, 225703 (2004). 